\title{
Scientific literature uses in Geography: indexing and "overlap" in four bibliographic tools
}

\section{ŞTIRBU Simona ${ }^{1}$,GRECO Ninfa ${ }^{1}$}

${ }^{1}$ Library of Sciences and Technologies, ULiege Library,

University of Liege, Belgium

\{Simona.Stirbu, N.Greco\}@uliege.be

\section{Aim}

Evaluate the interest for geographers to use Google Scholar (GS) over the commercial databases Web of Science (WoS), Scopus (multidisciplinary databases), and GeoRef (specialized in geosciences).

\section{Methods}

Searches in the four bibliographic tools (BT) of a sample consisting $\mathbf{7 2 0}$ citations of three dissertations (Belleflamme, 2015; Dubois, 2014: Trotta, 2014) presented in the ULiège (Belgium) Department of Geography during the $2014-2015$ academic year, respectively in Climatology, Tourism and Geomatics.

\section{Results}

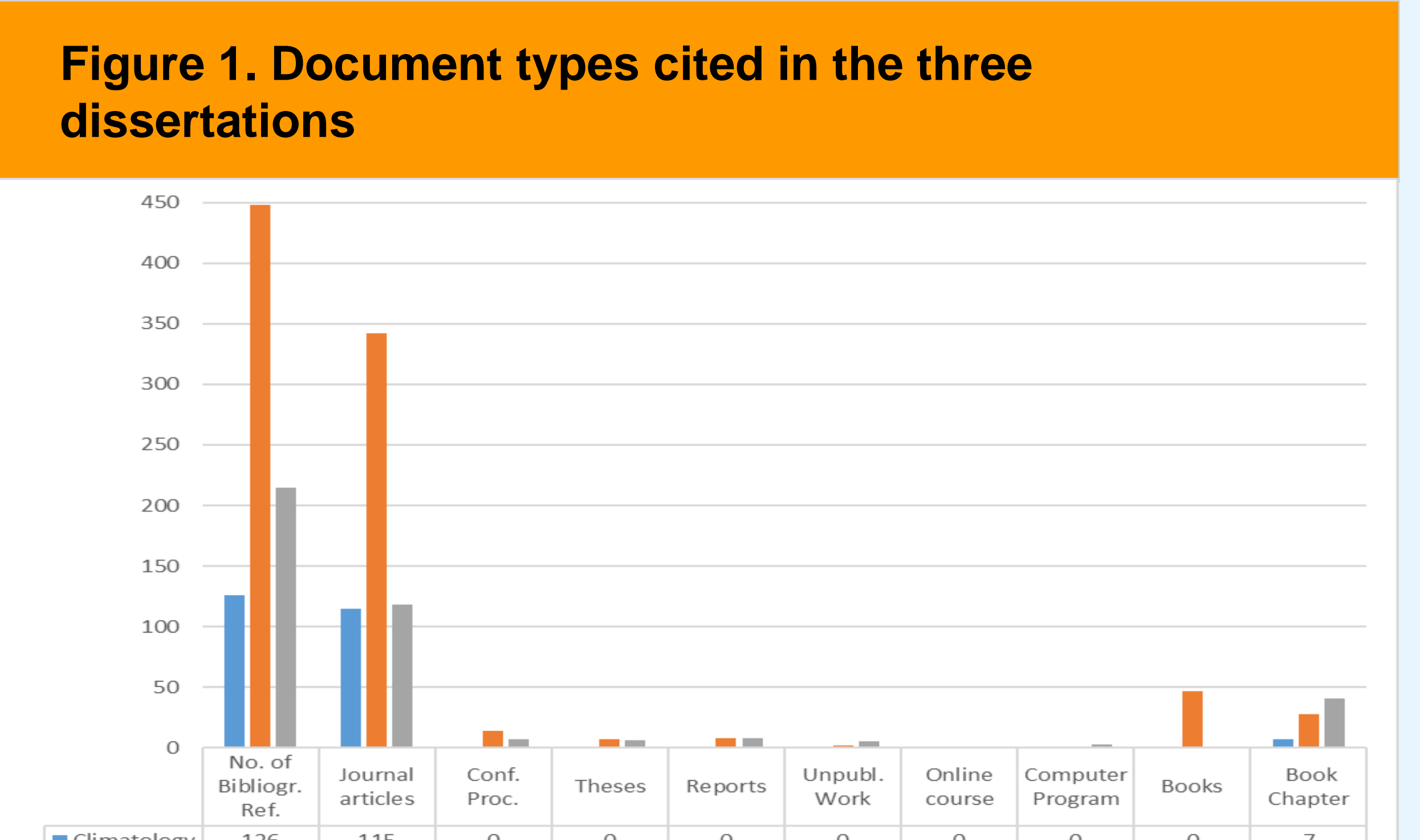

Figure 2. Bibliographic references by sub-discipline and $\%$ indexed in BT

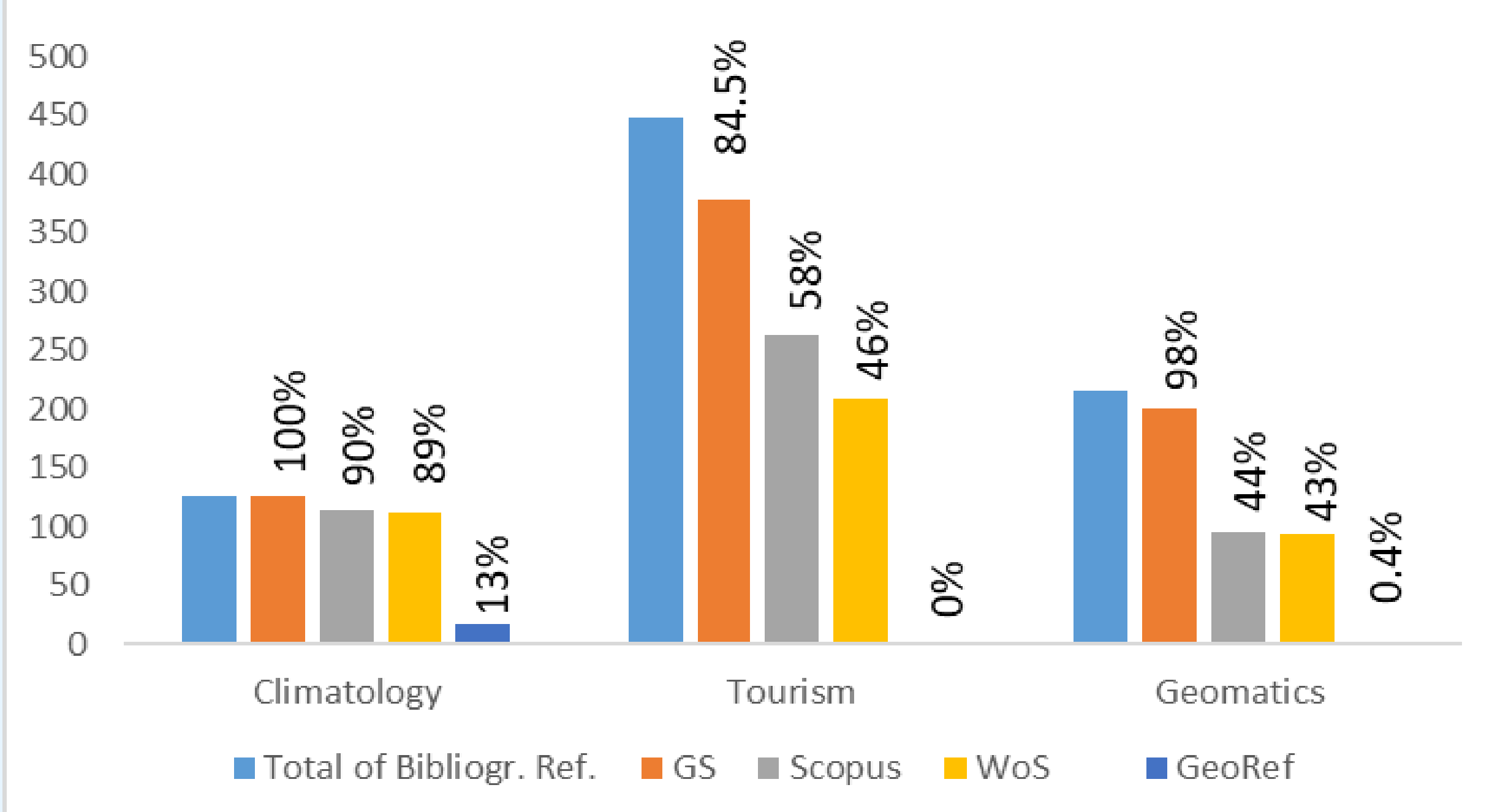

Figure 3. Journal articles by sub-duscipline and \% indexed in BT

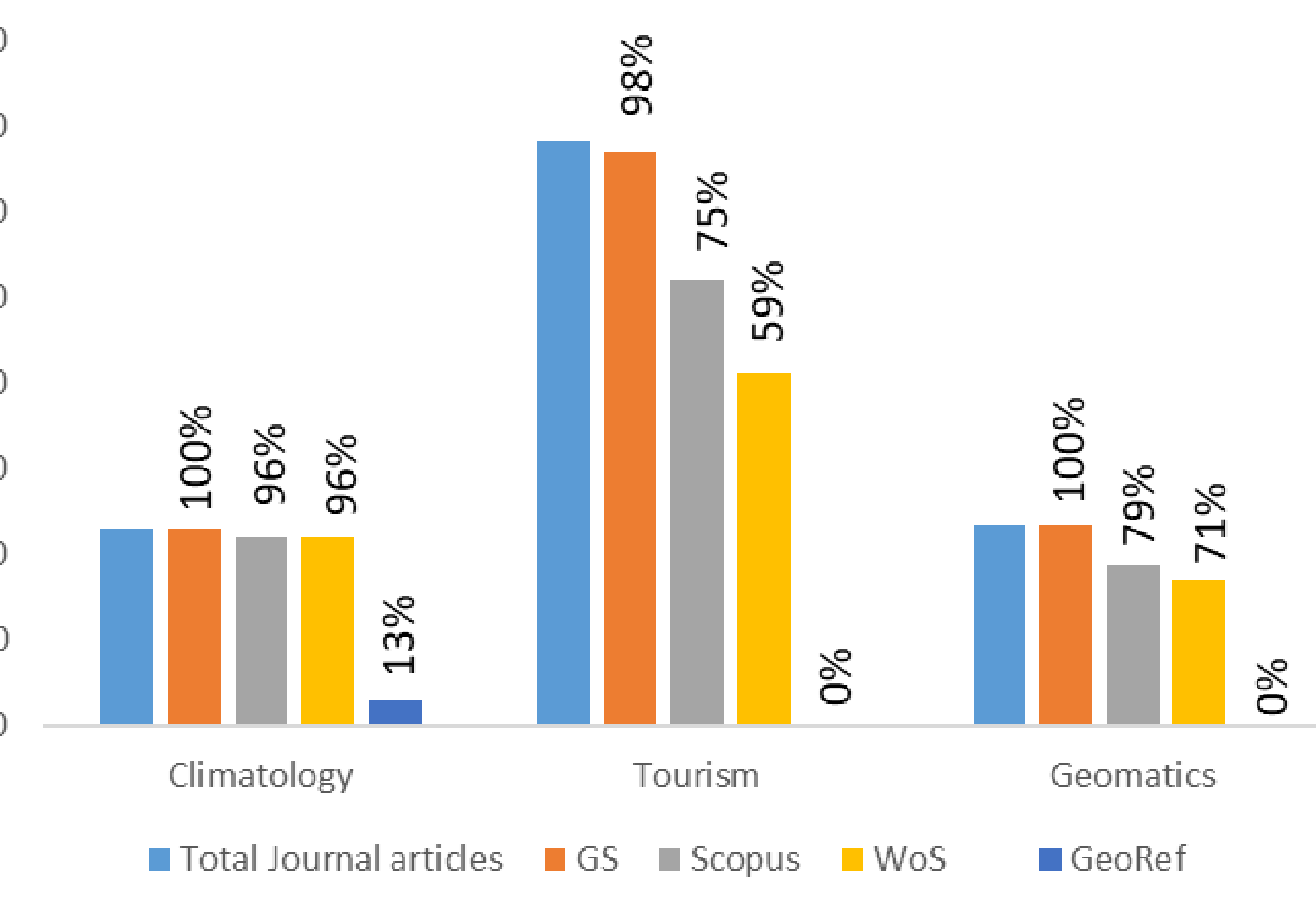

\section{"Overlap" and Venn Diagrams}

\section{Figure 2. Bibliographic tools overlap for the dissertation in Climatology}

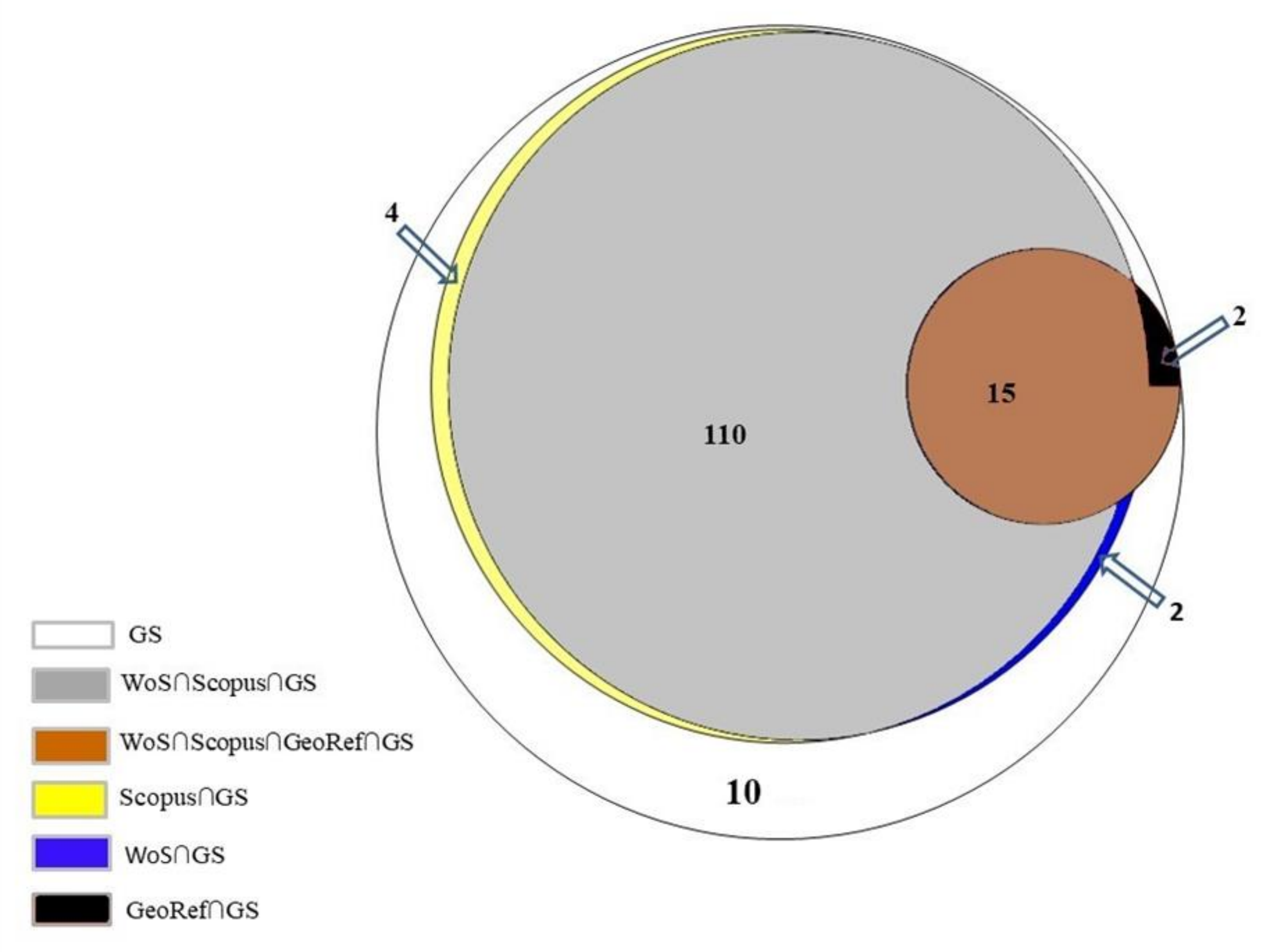

All the 126 references of the dissertation in Climatology are indexed by GS

-110 references are common to WoS, Scopus and GS

-GeoRef, retrieves only 17

references for this bibliography
GS retrieves 379 of the 448 references in Tourism which is the largest number of references among the bibliographic tools analyzed

198 references are indexed by all the tools, excluding GeoRef which does not index any reference for this bibliography

igure 3. Bibliographic tools overlap for the dissertation in Tourism

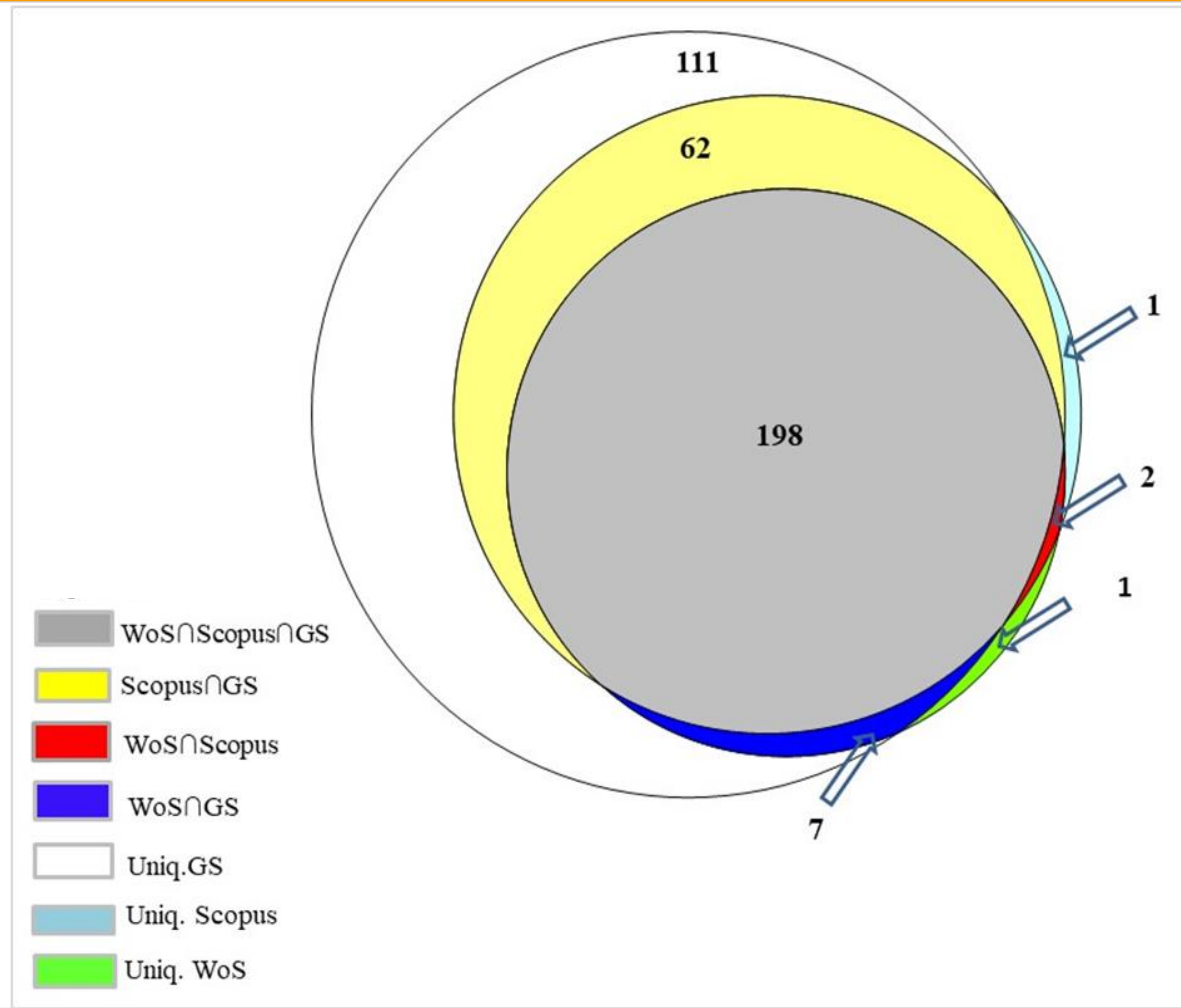

Figure 4. Bibliographic tools overlap for the dissertation in Geomatics

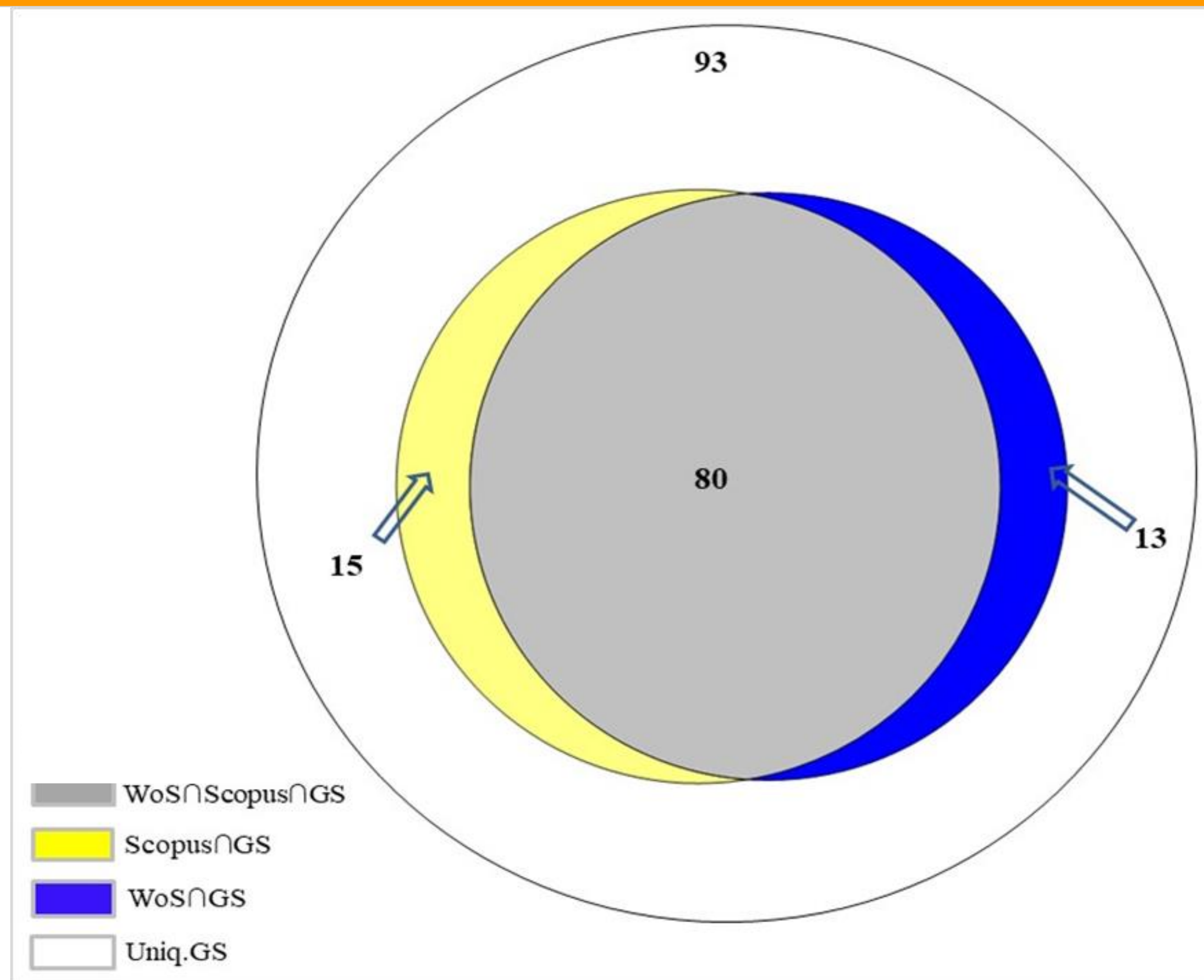

From a total of 215 references, GS retrieves 201 references -80 references are common to the three bibliographic tools, GS, Scopus and WoS, while GeoRef indexes only one reference (not represented on the diagram)

\section{It turns out that for the three} bibliographies, it is always GS that indexes an additional number of "unique" references, and recovers almost $100 \%$ of the other bibliographic databases.

\section{Findlings and Conclusions}

- In terms of indexed references, GS is the most comprehensive bibliographic tool, having found the majority of references searched with values close to $100 \%$ for the three bibliographies, all document types combined

- Considering the results and analyzes above, it appears that GS is a bibliographic tool that behaves very well when it comes to recover references indexed by traditional bibliographic databases

- Our results indicate a much higher potential for GS to find "unique" bibliographic references such as journal articles but also grey literature, books and book chapters, not indexed by commercial databases.

- More extensive analysis are needed to confirm our findings. 